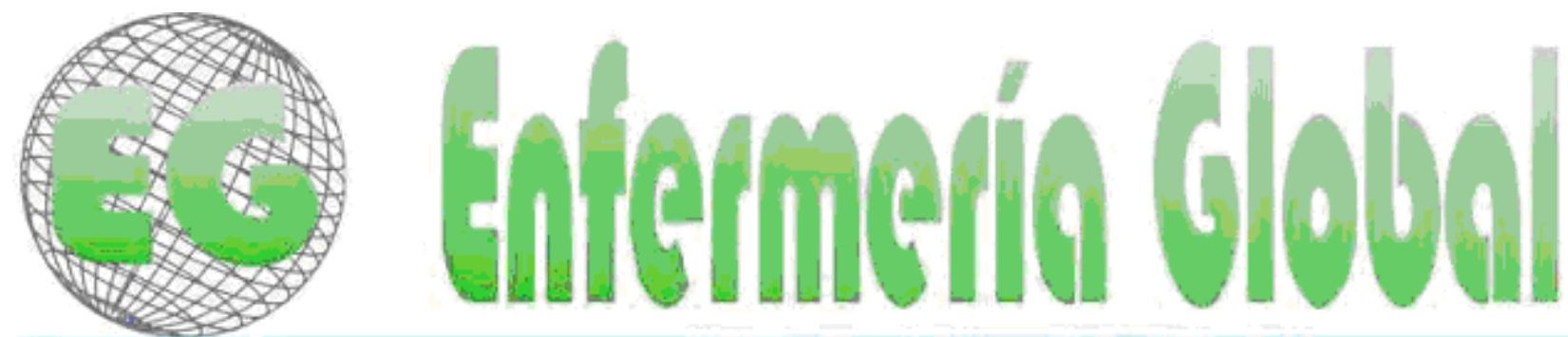

15SN $1696-6+25$

Revista electrônica cuatrimestral de Enfermeria

$N^{\circ} 19$

Junio 2010

www.um.es/eglobal/

DOCENCIA - FORMACIÓN

\title{
VIVENCIAS Y SIGNIFICADOS DEL PRIMER BAÑO DADO POR LA PUÉRPERA A SU HIJO RECIÉN NACIDO
}

AS VIVÊNCIAS E OS SIGNIFICADOS DO PRIMEIRO BANHO DADO PELA PUÉRPERA EM SEU FILHO RECÉM NASCIDO

*Souza Carvalhêdo, D., *Monteiro Lotufo, F., **Rodrigues da Silva

Barbosa, MA., "**Munhoz Gaíva, MA., "Lisboa, SR.

*Alumnas del Curso de Graduación en Enfermería. **Profesora Magister del Curso de Enfermería y Obstetricia ***Profesora Doctora del Curso de Enfermería y Obstetricia. Facultad de Enfermería de la Universidad Federal de Mato Grosso - Brasil.

(Trabalho de Conclusão de Curso apresentado ao Curso de Enfermagem e Obstetrícia como requisito parcial para a obtenção do título de Bacharel em Enfermagem pela Universidade Federal de Mato Grosso - Brasil.)

Palabras clave: Alojamiento conjunto; Recién nacido; Educación en salud

Palavras chave: Alojamento conjunto; Recém-nascido; Educação em saúde.

\section{RESUMEN}

Trátase de un estudio descriptivo-exploratorio de abordaje cualitativo, realizado en el alojamiento conjunto de un hospital escuela de Cuiaba-MT. Tuvo como finalidad comprender las vivencias y los significados del primer baño dado por la puérpera a su hijo recién nacido, en alojamiento conjunto, bajo supervisión de la enfermera. La colecta de los datos fue realizada a través de la observación participante y entrevista. Los resultados mostraron que deben implementarse nuevas prácticas relacionadas con la educación en salud para que las puérperas se sientan más seguras, respetadas en su singularidad y tengan mayor aprovechamiento en relación con los cuidados a su recién nacido.

\section{RESUMO}

Trata-se de um estudo descritivo-exploratório de abordagem qualitativa, realizado no alojamento conjunto de um hospital escola de Cuiabá-MT. Teve como objetivo, compreender as vivências e os significados do primeiro banho dado pela puérpera em seu filho recém-nascido, em alojamento conjunto sob supervisão da enfermagem. A coleta dos dados foi realizada através da observação participante e entrevista. Os resultados mostraram que novas práticas relacionadas à educação em saúde devem ser 
implementadas para que puérperas sintam-se mais seguras, respeitadas em sua singularidade e tenham maior aproveitamento relacionados aos cuidados com o seu recém-nascido.

\section{INTRODUCCIÓN}

Nacer era hasta hace algunas décadas, un acontecimento que ocurría en el ámbito domiciliario. Al lado de personas conocidas, la mujer sufría los dolores del parto y el bebé llegaba a este mundo en contacto con las personas que a partir de entonces formarían parte de su vida. Entretanto, la evolución socioeconômica, junto al perfeccionamiento técnico-científico en el área materno-infantil, hizo que las mujeres pasasen a ser asisitidas en instituciones hospitalarias. Así, ante la necesidad de minimizar los riesgos para la salud de la madre y del niño, tuvo lugar la implantación de nidos y, como consecuencia, la separación de la madre y del hijo durante el periodo de internamiento. Posteriormente, ante las investigaciones que señalaban los daños provocados en la relación madre hijo, se creó el Sistema de Alojamiento Conjunto que favorece la interacción y la participación activa de los padres en los cuidados al recién nacido.

El Sistema de Alojamiento Conjunto es definido, según el Ministerio de Salud, como un sistema hospitalario en el que el bebé sano, tras su nacimiento, permanece al lado de la madre las 24 horas del día, en un mismo ambiente, hasta el alta hospitalaria de ambos, lo que permite a los padres recibir orientaciones que los hacen aptos para prestar cuidados al hijo. Con ello se incentiva también el amamantamiento, el vínculo entre los familiares y se contribuye a la reducción de los índices de infección hospitalaria ${ }^{(1)}$.

Para el funcionamiento efectivo del Sistema de Alojamiento Conjunto, atendiendo a sus primeras finalidades, es necesario que el equipo de salud adopte una postura diferenciada, lo que exige conocimiento sobre las necesidades de su clientela, compromiso y dedicación en la asistencia prestada a la madre y al bebé. El modelo vigente de asistencia específica para la puérpera valora como fundamental los aspectos educativos orientados al desarrollo de habilidades maternas con la finalidad de instrumentalizar el cuidado de la mujer al hijo, responsabilizándola de este cuidado. Especialmente en las instituciones públicas, la mujer no opina sobre la asistencia que se le presta o la modalidad de ingreso a que es sometida, sin cuestionar siquiera la atención recibida ${ }^{(2)}$.

El desarrollo de este estudio se basó en la observación inicial de que las puérperas experimentaban diversos sentimientos al dar el baño a su hijo recién nacido, sobre todo la primera vez, en presencia del equipo de enfermería. En el hospital donde se realizó este estudio, la demostración del baño del recién nacido a la puérpera se implantó desde su creación y continúa efectuándose en el alojamiento conjunto del hospital según la siguiente secuencia:

El recién nacido es envuelto en una toalla de modo que solo asome la cabeza, se protegen los oidos con los dedos pulgar y anular, realizándose la higiene de la parte externa de los ojos, rostro y cuero cabelludo. Terminada la higiene del cabello se seca inmediatamente y las orejas y la nariz se limpian con una toalla fina y suave. A continuación, se le desnuda totalmente, sumergiéndole en una bañera con agua tíbia para la higiene de otras partes del cuerpo según la secuencia: región cervical anterior, tórax, miembros superiores (MMSS), abdomen, zona umbilical, genitales, 
miembros inferiores (MMII), región dorsal y nalgas. Una vez terminado el baño, el recién nacido es secado y colocado sobre superficie seca, envuelto en toallas secas y sometido a cura del cordón umbilical con alcohol al 70\%. Después es vestido y si es necesario reconfortado.

Esta técnica con su recién nacido se enseña individualmente a las puérperas el primer dia tras el parto, permaneciendo ellas como observadoras solamente, comunicándoles que al día siguiente deberán hacerlo ellas ante el equipo de enfermería que en esta ocasión asumirá el papel de observador. Ello se hará en la enfermería y el equipo aprovecha el momento para además de certificar que la puérpera ha aprendido esta técnica, reforzarla o corregirla caso de que sea necesario.

Hay que destacar que el tipo de orientación utilizado por el equipo de enfermería es llamada de demostración y puede ser adoptada para la orientación individual o grupal, siendo más utilizada en las maternidades en el alojamiento conjunto. Este tipo de estrategia educativa es ideal cuando se pretende comunicar una determinada información y mostrar procedimientos ${ }^{(3)}$. Por ello, en esa situación puede usarse asociada a otras estrategias que faciliten más lo aprendido, sobre todo en la fase especial en que se encuentran, es decir, el puerperio.

En un estudio ${ }^{(4)}$ que analizó una práctica educativa en la que se utilizó un juego educativo a base de preguntas para discusión en grupo de puérperas con temas relacionados con el amamantamiento y cuidados con el recién nacido, se constató que la estrategia utilizada contribuyó al aumento del conocimientos sobre los temas citados. Entre otros puntos positivos, el uso del juego estimula el proceso intelectual, promueve la interacción y desarrollo entre los participantes, estimula el interés y aporta elementos para un cambio de actitud.

Este estudio tuvo como objeto de investigación las vivencias y los significados del primer baño dado por la puérpera a su hijo recién nacido en alojamiento conjunto bajo la supervisión del equipo de enfermería. El objetivo del mismo fue comprender los significados y vivencias de la puerpera relacionados con el primer baño dado por ella a su hijo recién nacido.

\section{METODOLOGÍA}

Trátase de una investigación descriptiva exploratoria, a la luz del abordaje cualitativo, pues esta trabaja con significados, motivos, valores, actitudes y aspiraciones, posibilitando el estudio de las relaciones, procesos y fenómenos que no pueden ser reducidos a operacionalización de variables ${ }^{(5)}$, como en el estudio en cuestión.

Este fue realizado en el alojamento conjunto de un hospital público de Cuiabá Mato Grosso. En él participaron un total de 17 puérperas, con edades que varían de 16 a 29 años, de las que 8 tuvieron parto normal y 9 parto cesárea, 8 primíparas y 9 multíparas. Los criterios básicos de selección de los sujetos fueron la permanencia en el sistema de internamiento conjunto de la unidad seleccionada, en el intervalo de tiempo de la colecta de datos, y la aceptación de participación en la investigación. 
La recogida de datos fue realizada a través de la observación y de la entrevista que tuvo como cuestión norteadora la siguiente pregunta: ¿Cómo fue para usted el haber dado el primer baño a su hijo recién nacido?

Definimos como fuente de información básica, el momento del primer baño que la puérpera dio a su recién nacido bajo la supervisión de enfermería y el contexto relacional presente en ese momento, abarcando a la puérpera, al recién nacido y a los trabajadores de enfermería, sumado al discurso específico de las puérperas acerca de determinados procesos que las rodearon.De modo complementario, el discurso del equipo de enfermería se utilizó para confirmar algunas observaciones efectuadas. Todas las entrevistas fueron realizadas en el propio hospital, a continuación de la realización del baño hecho por la puérpera, y anotadas manualmente en el momento de las mismas.

Los datos derivados de las observaciones y entrevistas fueron codificados y agrupados por su semejanza, constituyéndose en subtemas que a su vez formaron temas más destacados. De ese proceso, surgieron cuatro grandes temas que fueron objeto de esta investigación: El miedo a dar el baño al recién nacido, el nerviosismo y la inseguridad al dar el baño al recién nacido, las relaciones del equipo de enfermería durante el baño que la madre ejecuta en su hijo.

Siguiendo las orientaciones de las normas que reglamentan investigaciones en seres humanos ${ }^{(6)}$, el proyecto fue aprobado bajo PARECER oficio $s / n$ fechado el 22 de enero de 2004 del Comité de Ética en Pesquisa del hospital en cuestión y los sujetos entrevistados concordaron y firmaron término de consentimiento informado en la investigación. Por respeto a las cuestiones éticas los sujetos fueron identificados a través de pseudónimos.

\section{PRESENTACIÓN DE LOS RESULTADOS}

\subsection{El miedo a dar el baño al recién nacido}

Según el diccionario, MIEDO es un sentimiento de viva inquietud ante la noción de un peligro real o imaginario, de una amenaza; susto, pavor, temor terror, recelo ${ }^{(7)}$.

Este sentimiento se tradujo en la dificultad de acomodación del recién nacido en los brazos y en la bañera; en la manera de tocarlo (solamente con la punta de los dedos y rápidamente); durante la higiene de algunos segmentos como el cordón umbilical y los genitales, sobre todo cuando pertenece al género femenino. Percibimos que esto sucede en especial con las prímiparas mas no es exclusivo de éstas, las multíparas también relatan esas sensaciones al prestar cuidados, considerando el tamaño del recién nacido como un factor generador de miedo:

... y ella también es tan pequeñita que me da miedo de que se caiga y se dañe. (Rosa).

Ah, mi problema es solo el miedo, tengo miedo! [ ] Miedo de dañarle porque es muy pequeño (Jasmim). 
La fragilidad del bebé puede ser un hecho que asuste a las madres. En ese contexto se sienten con mucho miedo a equivocarse y extremadamente inseguras de que al menor desliz, suceda algo trágico; por eso el miedo a asumir los cuidados con el recién nacido es menor para las madres que tuvieron alojamiento conjunto, donde recibieron entrenamiento para lidiar con los pequeños problemas rutinarios y tuvieron oportunidad de empezar a conocer mejor al hijo ${ }^{(8)}$.

Acreditamos que ese sentimiento de miedo puede reducirse si se proporciona a la madre una experiencia de contacto físico con su hijo, de modo placentero y sin presiones, pues de lo contrario ese sentimiento se puede agravar, es decir, no es solamente la posibilidad de la madre de desarrollar cuidados directos al recién nacido lo que la convierte en una persona competente para cuidarlo, sino también, entre otros factores, la forma como el equipo la prepara para ese cuidado. Si esto se hace atendiendo también a las necesidades de la madre, percibiéndola como ser único, respetando sus sentimientos ante los cuidados al hijo, ciertamente los resultados serán mejores.

El miedo también es percibido en la manera como la madre toca a su hijo durante el baño:

...la madre tras haber cogido a su hijo se aproxima a la bañera mojando levemente su mano en el agua y llevándola lentamente para lavar con mucha delicadeza la cara de su bebé. (Nota de Observación)

El toque es una de las formas de establecer el vínculo madre e hijo y hay un periodo tras el nacimiento durante el cual se establece. El toque de las madres sigue un patrón bien definido: las madres tocan las extremidades de la criatura y después la cabeza, primero con la punta de los dedos y finalmente con la palma de la mano. Este periodo es crítico para el desarrollo de una interacción sana y vínculo normal ${ }^{(9)}$.

El toque es el siginificado humano, es dar vida a aquel que es tocado. Mas es preciso aprender/saber tocar con firmeza delicada, pues el bebé puede notar la inseguridad del toque materno ${ }^{(10)}$. El sentido del tacto también se desarrolla desde la época de gravidez y tiene un papel importante en la adaptación del recién nacido al mundo extrauterino, los primeros meses después del nacimiento pueden ser considerados como continuación directa del estado intrauterino, siendo necesario mantener el íntimo contacto corporal con la madre, para ello el bebé debe ser tomado en brazos con seguridad, debe ser amamantado a intervalos regulares, ser protegido, acariciado, precisa de que se le hable, de que le tranquilicen ${ }^{(10)}$.

La rapidez con que el bebé reacciona a la ausencia de contacto seguro se constata cuando se le deja sobre una superficie plana como una mesa, sin apoyo de la mano. Él reacciona inmediatamente con susto y llanto. Las madres nerviosas acostumbran cuando toman al niño, a cogerlo sin firmeza o de modo inseguro. Así, la inseguridad de la madre es captada por el niño. La piel es el órgano de recepción sensorial fundamental para el desarrollo humano. El bebé capta mensajes con sus receptores localizados en las articulaciones musculares, de manera que las presiones ejercidas sobre su piel le dicen lo que siente por él aquella persona que lo está acariciando ${ }^{(10)}$.

Creemos que si no se dan las condiciones a las madres para que el proceso de interacción inicial fluya de manera que se sientan seguras, ella puede transmitir a través de su piel/toque todos los sentimientos perjudiciales a su bebé y este reaccionará negativamente a la madre por un principio de acción reacción, 
provocando en ambas partes reacciones cada vez más fuertes y conflictivas lo que puede causar daños al proceso interactivo madre-hijo.

Aseguramos que el equipo que se enfrenta a la clientela de alojamiento conjunto precisa saber que ese contacto es un proceso creciente de interacción madre-hijo y debe proporcionarse de manera delicada, segura y que los trabajadores de enfermería deben estar preparados para contribuir de manera positiva, o sea, favoreciendo un ambiente seguro y sin conflictos para que la puérpera y su hijo consigan establecer esa interacción de manera efectiva. Entre tanto, si el equipo no se concienza de la importancia de ese proceso, puede estimular en la madre el miedo a aproximarse y cuidar del bebé, generando sentimientos semejantes al pánico:

... no puedo, [ ] no puedo porque tengo miedo (Margarida).

Después de la conversación con esta puérpera sobre la necesidad de que ella aprenda a dar el baño a su hijo, ella aceptó el realizarlo y tras el procedimiento nos dijo en una entrevista ya un poco aliviada:

Ah, fue difícil, tengo que dar el baño más rápido, mas, yo tenía miedo, creía que no lo iba a conseguir! (Margarida).

En el alojamiento conjunto, la educación para la salud debe ser el principal foco de acción del equipo posibilitando que la puérpera obtenga conocimientos y habilidades para la realización del cuidado con el bebé y con ello disminuya su ansiedad en el desempeño del papel de madre ${ }^{(11)}$. El miedo a asumir los cuidados del bebé fue menor en las que estuvieron en alojamiento conjunto, donde recibieron algún entrenamiento para afrontar los pequeños problemas rutinarios y tuvieron oportunidad de empezar a conocer mejor a su hijo.

Aunque algunas madres sienten miedo, para otras la experiencia en el cuidado de otros hijos convierte el momento del baño en un acto placentero:

Fue bueno, ya estoy acostumbrada, ya bañé a dos, mas, bañar a este de aquí también fue bueno! (Orquídea).

Por tanto, la novedad de los sentimientos no se limita a la primera experiencia de tener un hijo. Sin duda, a partir de la segunda gravidez, ya se conoce una serie de sentimientos y vivencias propias de la situación, ya se pasó por la experiencia de un primer parto, tal vez ya se está con más confianza en la capacidad de lidiar con un bebé pequeñito ${ }^{(6)}$. Tanto la multípara como la prímipara pueden experimentar sentimientos semejantes en la experiencia de tener y cuidar de un hijo recién nacido y que la maternidad es una experiencia singular, esto es, cada mujer la vivencia de una manera única e intransferible, independientemente del número de hijos que haya tenido.

El sentimiento de miedo también se presenta em lo que se refiere al lavado e higienización del cordón umbilical durante el baño:

Fue difícil porque tuve miedo de lavar el ombligo y hacer el curativo (Rosa). 
Algunos estudiosos ${ }^{(12)}$ recomiendan que no se debe lavar la zona del cordón durante el baño del recién nacido por el peligro de infección. En la institución donde se hizo ese estudio se estableció por rutina el lavado del cordón con agua y jabón durante el baño, lo que suscita en las madres dudas y miedo a proceder conforme a esa técnica, pues la práctica corriente en algunas comunidades es que el cordón umbilical sólo debe ser lavado tras su caída. Se cree que si este se moja puede infectarse lo que dificultaría su cicatrización o hasta incluso provocar el tétanos neonatal.

Según orientaciones técnicas de la Secretaría de Salud del Estado de São Paulo, se aconseja lavar con agua y jabón, secar y limpiar el cordón umbilical con algodón empapado en alcohol al $70 \%$, que por su rápida acción antiséptica, sin efecto residual, acelera el secado y la caída ${ }^{(13)}$. En nuestras observaciones en este estudio percibimos que la mayoría de las mujeres puérperas tienen miedo a manipular el cordón umbilical y no realizan correctamente su higiene durante el baño.

Después de tomado, la madre mantiene al recién nacido desnudo para iniciar el baño, el mismo se encuentra con el cordón umbilical envuelto en gasa, la madre va y pregunta: "¿puedo mojar el ombligo?" La funcionaria volviéndose, observa y dice: "puede lavar derechito el ombligo, usted está con miedo mas puede lavar" (Nota de observación).

El miedo a manipular el cordón umbilical está presente en todas las madres de este estudio y hace que muchas se sirvan de algunas estrategias para luchar con ese sentimiento y una de ellas es la de "ignorar" la existencia del mismo durante el baño:

... no lava el cordón, lava los genitales superficialmente, gira al recién nacido, lava las costillas y región anal, coloca al recién nacido en la cama y comienza a secarlo. (Nota de Observación).

A veces, la enfermería percibe este comportamiento materno y lo trata con mucha objetividad, sin tener en cuenta los sentimientos maternos:

...la puérpera comienza lavando con el rascador los piececitos y cuando llega al abdomen del recién nacido, la funcionaria dice: "puede ir mojando ese ahí y tirando" (se refiere al curativo de gasa enrrollado en el ombligo,) la puérpera encoge la cabeza y nos escucha con expresión de miedo, la funcionaria repite: "ipuede tirar!", como la puérpera no tuvo la iniciativa de tirar del curativo, la funcionaria se aproxim y lo retira. En ese momento la puérpera desvía la escucha, la colega de enfermería dice: "parece que va a doler" y la madre asiente con la cabeza. (Nota de observación).

En ningún momento la enfermería aclara que el área en cuestión es indolora, por la falta de inervación local, lo que puede ante la falta de conocimiento materno aumentar todavía más su miedo, principalemente en los casos arriba relatados.

De este modo, la distancia entre el conocimiento científico y el sentido común se mantiene en el interior de los servicios de salud, perpetuando así la relación infructuosa entre el profesional y el cliente, impidiendo el intercambio de conocimiento y el alcance de una asistencia de calidad ${ }^{(3)}$. 
Un estudio sobre la representación de la puérpera sobre el sistema de alojamiento conjunto revela que el hecho de que las puérperas aclaren sus dudas durante el internamiento por los profesionales que les asisten es percibida como una forma de ayuda y se constituye en elemento cualificador de la asistencia y de reelaboración de su representación acerca de la hospitalización ${ }^{(14)}$.

Acreditamos que el equipo debe justificar, a través de conocimientos teóricos, algunas de sus prácticas y afirmaciones ante las puérperas, para que ellas se sientan más seguras y menos afligidas ante algunas acciones de enfermería y no únicamente decirles de forma imperiosa lo que tienen que hacer. Ofrecer informaciones simples, con justificativas claras, es una de las estrategias que se puede utilizar para que las puérperas se sientan más seguras para desarrollar los cuidados con sus hijos y para que ellas adquieran confianza en el profesional que las orienta.

\subsection{El nerviosismo y la inseguridad al dar el baño al recién nacido}

Cuando se desarrolla una tarea por primera vez y en presencia de otros que la observan se experimentan en alguna medida incomodidades que forman parte de las experiencias de las madres quienes durante la constatación de su aprendizaje por miembros del equipo de enfermería pierden la voluntad poniéndose nerviosas:

\section{Me puse nerviosa por estar siendo observada (Tulipa).}

El equipo en cuestión, al adoptar una actitud disciplinada utiliza la visión jerárquica que es uno de los instrumentos del poder disciplinario ${ }^{(15)}$. El mirar jerárquico se concretiza en la institución a través de procesos como la supervisión que el equipo de enfermería dirige a las puérperas durante el procedimiento del primer baño. Ese mirar a través del cual se hace la supervisión y control de las actitudes de ellas durante el desarrollo de la técnica es un dispositivo disciplinar para inducir determinados comportamientos relacionados con los cuidados con el hijo.

Un estudio realizado en un hospital universitario sobre el comportamiento del recién nacido durante el baño reveló que el primer baño de la puérpera a su hijo acompañada por una auxiliar que participa como instructora, corrigiendo cuando la puérpera hace algún procedimiento incorrecto, provocaba que esta estuviese más preocupada con la secuencia correcta que con el propio bebé. Además la puérpera se mostraba irritada y cansada al terminar el baño y el bebé pasaba la mayor parte del procedimiento agitado o llorando. Al observar el segundo baño, ya sin el acompañamiento de la auxiliar se constató que a pesar de existir todavía preocupación con la secuencia del baño, las puérperas reaccionaron mejor ante los cambios comportamentales de su hijo. Así las madres llevaban a los bebés de estados de agitación a estados de más calma, facilitando la interacción entre ellos ${ }^{(16)}$.

El nerviosismo materno se manifesta también por otros motivos. Algunas madres se ponen nerviosas con el llanto del recién nacido durante el procedimiento y caso de tener experiencias con otro recién nacido tampoco disminuye ese sentimiento:

Quedé angustiada y aprensiva cuando el nene comenzó a llorar, con empeño de acabar inmediatamente. A pesar de haber dado ya el baño a otros nenes quedé un poco nerviosa! (Dália). 
El lloro es un lenguaje, es comunicación. Es el principal recurso de que el bebé dispone para comunicar sus necesidades y expresar lo que siente. El influjo del llanto del niño sobre los adultos que cuidan de él varía considerablemente desde despertar ternura hasta la angustia de impotencia total, llegando a la rabia traducida en el deseo de huir para no tener que continuar escuchando aquel llanto tan difícil de entender. En los primeros meses de vida del bebé es difícil saber la causa del llanto pero poco a poco las personas que cuidan del bebé van aprendiendo a distinguir las melodías de los diferentes tipos de llanto y lenguaje corporal. Para ello es necesario que las personas tengan sensibilidad, paciencia y atención cariñosa, para gradualmente conocer al bebé y viceversa. Este proceso puede ser más fácil a partir del segundo hijo, pues la práctica de cuidar del primer hijo ayuda mucho cuando los otros nacen ${ }^{(17)}$.

El miedo a no saber cuidar puede atenuarse cuando los padres deciden hundirse en las raíces no verbales de la comunicación, en los aspectos sensoriales del contacto piel a piel con el bebé, navegando amorosamente en el escuchar, en la sensibilidad y en la intuición, procurando conocer las características del hijo, sus ritmos, sus estados de humor, la riqueza de su desarrollo ${ }^{(17)}$.

Según lo dicho anteriormente, percibimos que el llanto del bebé parece estar relacionado con la incomodidad del baño, del agua. Esa incomodidad parece haber sido percibida por la puérpera aunque no consiguió localizar la causa exacta de la misma.

Así, la reacción de los padres ante el llanto del bebé varía en una gama amplia de sentimientos, desde la preocupación hasta la irritación o la desesperación de llorar junto con él (17). Aunque hasta que estos sentimientos no son percibidos por el equipo no se configuran como objeto de atención.

La puérpera coge al recién nacido por el cuello, está con las manos temblando, mira a la funcionaria que le enseña como asegurar al bebê, tapándole los oídos para que no entre agua (Nota de observación).

Si bien algunas madres, a pesar del nerviosismo, cumplen la tarea en ellas delegada, hay otras que se niegan a desarrollarla de manera firme y determinada, no cediendo a las órdenes de la funcionaria:

¡Yo no voy a dar el baño, estoy nerviosa, no lo voy a dar! (Azaléia).

Así, muchas se arrogan el derecho de no desarrollar los cuidados para los que están siendo solicitadas, enfrentándose de manera explícita a la presión del equipo de enfermería.

Respecto a la inseguridad, esta se hace presente cuando la madre se muestra con conocimientos que no poseía anteriormente, a pesar de haber constatado que la manera como fue orientada en el hospital era mejor que la que desarrollaba con otros hijos:

Yo no sentí seguridad a decir verdad, yo no sabía que tenía que ser así, cubrir al bebé primero, los otros hijos que yo tenía los colocaba 
directamente en la bañera, pero así es más fácil, el bebé llora menos, mas yo preciso de más información. (Papoula).

Por esto se hace necesario que el equipo que actúa en esa unidad comience a pensar en una práctica educativa más participativa que considere y valore el saber anterior de esas mujeres y les dé la oportunidad de situarse como sujetos con potencialidades y conocimientos previos, para humanizar la asistencia, disminuyendo los conflictos, a través de una relación equipo-cliente lo más horizontal posible.

La valorización del conocimiento que el individuo posee se hace imprescindible para iniciar el proceso de promoción de su salud en el ámbito de la relación pofesional/cliente ${ }^{(18)}$. Así, cuando se trata de educación para la salud, el requisito básico para su establecimiento es la capacidad de los profesionales de establecer relación de confianza con el cliente, para que este acepte el conocimiento transmitido, al mismo tiempo que sustituye conceptos erróneos u obsoletos referentes a las prácticas del cuidado en salud ${ }^{(3)}$. .

La educación como proceso histórico evolucionó a medida que la sociedad evolucionó. Es un proceso reflexivo, dinámico, complejo, social, reflexivo, terapéutico y ético que se construye a partir de las interacciones entre los seres humanos. Quien enseña, aprende, y quien aprende, enseña; intercambiando conocimientos y experiencias mutuas, una vez que cada ser interactúa el hecho con sus ideas, sus valores, sus creencias actitudes y experiencias ${ }^{(19)}$.

Es preciso comprender que una educación dirigida al cuidado humano no es aquella que modela o impone determinadas reglas, o que entrena: es una educación que permite el llegar a ser, el responderse ${ }^{(20)}$. Cuidar abarca la competencia y la confianza. Por competencia se entiende el estado de tener conocimiento, juicio, habilidades, energía, experiencia y motivación necesaria para responder adecuadamente a las demandas de las responsabilidades profesionales. La confianza se refiere a la cualidad que promueve relaciones en que las personas se sienten seguras y respetadas ${ }^{(21)}$.

\subsection{Las relaciones establecidas durante el baño}

Los preparativos para el baño de los recién nacidos se inician cuando las mujeres hacen la primera comida del día, en torno a las 07 horas; a pesar de que el equipo hace una llamada o aviso, no se excluye la intención de la enfermería de concluir sus tareas en tiempo hábil:

La funcionaria pasa por la enfermería y dice: estamos esperando a que ustedes coman para comenzar a dar los baños (Nota de observación).

Esta actitud denota la rigidez y la disciplina en el ambiente hospitalario. Sabemos que la disciplina en términos de organización en un ambiente hospitalario es necesaria para la optimización del tiempo, mas en algunos momentos debería haber flexibilidad en el desarrollo de algunos procedimientos como el baño del recién nacido. Esa inflexibilidad constatada en este estudio, nos hace interrogarnos en qué medida ello es saludable para las relaciones entre las puérperas y el equipo de enfermería. 
La presión también aparece relacionada en lo que dice respecto a la capacidad de ejercer tareas maternas como si fuese algo natural que se presenta de manera espontánea en cuanto la mujer se hace madre, es decir, la idea de que la mujer al hacerse madre debe comportarse como una verdadera madre respondiendo al modelo construido sobre ello en su espacio social. La respuesta a ese modelo socialmente construido en este contexto parece depender de su estado físico, de su dependencia del dolor, y cuando ella no corresponde a esa expectativa, se duda de su compromiso relacionado con los cuidados del hijo:

"no adelanta, tiene madre que es así, resistente, no adelanta ni habla, nosotros ya enseñamos. Ayer habló que estaba con dolor (refiriéndose a la puérpera), todo bien, cesárea todavía duele, mas ya dice que la tienen que levantar, cuanto más esté parada es peor, sólo que ella no quiere cuidar del hijo!" (Nota de observación).

En nuestra sociedad todavía circula en determinados segmentos que la maternidad debe considerarse placentera, independientemente de las condiciones físicas o sociales que la mujer tenga para ejercerla. Cuando se encuentra el perfil opuesto al de "buena madre", este no se acepta fácilmente y ,con frecuencia, se niega como apropiado a una "madre" (22).

Así el mito de la buena madre es un mito profundamente enraizado y de algún modo perjudica a las mujeres, pues se sobreentiende que la madre puede ser una buena madre. La madre ideal debe ser notada, toda maternal, olvidándose de sí para solo pensar en sus hijos ${ }^{(23)}$. El mito de la maternidad aceptado en nuestra sociedad afirma que las madres sienten amor por sus hijos y les tienen tiernos sentimientos, consecuentes al acto biológico de la procreación. La idea de que con el nacimiento de los hijos las mujeres se vuelven más abnegadas y generosas, diferentes de cómo eran antes, experimentando la satisfacción suprema de sacrificarse por sus hijos; las madres quedan exentas de ansiedad y pasión, representando la pureza de las madres al servicio del hijo ${ }^{(24)}$.

La supervisión para que las madres desarrollen la técnica del baño se hace de forma agresiva con gestos y actitudes groseras y en forma de devolver la técnica por parte de la puérpera a los funcionarios. De este modo, el cuidar se convierte en algo difícil e inadecuado cuando ocurre en situaciones que caracterizan solo el desempeño de una tarea:

¡Usted no está prestando atención! Mañana yo voy a ver y voy a vigilar y escuchar, yo vigilo lo que veo. Usted no presta atención, lo hace todo mal, la gente enseña, enseña y lo hace mal (Nota de observación).

Así la supervisión se vuelve agresiva en determinadas situaciones, evidenciándose varias faltas de respeto en lo que se refiere a las limitaciones físicas de la puérpera, a su inexperiencia en bregar con el recién nacido al ritmo propio de cada una para dar cuenta de esa actividad y la imposición del "ser madre" en el imaginario popular.

Tales comportamientos pueden causar daños en una relación madre hijo que está empezando a formarse y en la capacidad maternal que la mujer está intentando construir. En esas situaciones, pueden presentarse algunos problemas emocionales 
relativos al ejercicio de la maternidad, pues el equipo no da oportunidad para que la puérpera presente sus dificultades y necesidades. Las exigencias que se piden a las madres según se espera de ellas, hacen que muchas de estas madres se vean en dificultades para llevarlas a cabo.

En el ámbito hospitalario, muchas veces por la necesidad de cumplir ciertas rutinas, hasta la enfermera ignora las condiciones físicas, emocionales, la falta de destreza/agilidad de las mujeres atendidas. Además, la percepción que se tiene de la maternidad y del ser madre contribuye a que la supervision sea más intensa, quedando claro el uso del poder:

"madre ¡moje esa mano derecha!, no va a lastimar al bebé. Pon un dedo en un oído y otro en el otro para que no entre agua", la puérpera intenta argumentar: "mi mano es corta". La enfermera dice: "si ya es madre mi hija, jtiene que dar cuenta!" (Nota de observación).

La puérpera comienza a enjabonar la cabeza del recién nacido sólo con la mano mojada, sin irrigar el cuero cabelludo del recién nacido, la enfermera dice: "niña, moja eso bien, frota con el jabón y restriega", la puérpera escucha y vuelve a lavar la cabeza sin maña, la enfermera continúa "restriega rápido!"[ ] La madre se pone a enjuagar la cabeza para retirar el jabón. La enfermera demostrando haber perdido la paciencia dice: "no hables y sé rápida"! [ ] "madre ahora usted lava debajo del cuello, la barriga, genitales y las piernas, enjuágale y mueve así su brazo, (muestra) lava las costillas, el pompis, y tiene que ser rápido, no puede quedar alisado no!" (Nota de observación).

No hay poder sin potencial de vuelta, o sea, el poder sólo se ejerce cuando hay resistencia/potencial de vuelta ${ }^{(15)}$. De este modo, al ser preguntada sobre el significado del primer baño, esta puérpera responde:

"¡Ah, es difícil! Más despacio yo lo cojo mejor, me puse nerviosa mas, ya, ya, yo aprendo, aprendí otras cosas, esto también voy a tener que aprender!" (mira a sus compañeras de enfermería y ríe mucho) (Violeta).

Ese comportamiento nos pareció una revancha por la situación que pasó, o sea, ella también utilizó el poder, pues fue fuertemente criticada, exigida por la enfermera de la unidad para la realización del baño a su hijo, ante todas las compañeras de sala.

La crítica es una forma de comunicación que suele ser nociva porque estimula el resentimiento y puede dificultar el vínculo. También perjudica la autoestima de la persona, especialmente cuando ella atraviesa fases de cambio en que se encuentra emocionalmente más vulnerable.

Conviene recordar que en especial en el primer hijo, la identidad de la mujer como madre es tan recién nacida como el bebé: frágil, vulnerable, sensible. En un período como este, la crítica es particularmente nociva, especialmente si viene de personas consideradas relevantes, toda vez que tiende a aumentar los sentimientos de autodesvalorización, inferioridad y falta de confianza en sí misma ${ }^{(25)}$. 
Además, la conducta fría, impersonal y puramente técnica en la atención es obviamente contraria a la actitud clínica: no nos permitimos, por razones diversas entrar en contacto con la persona que estamos atendiendo, sino que la encaramos como un objeto y nos conportamos como las máquinas, instrumentos y aparejos que utilizamos. En este modo de atender, el potencial de acción benéfica del profesional se reduce mucho. Como sucede con el uso de formas nocivas de comunicación, atender de modo impersonal acarrea bloqueo o estrechamiento de los canales de comunicación. A medida que no se crea el espacio de disponibilidad, el cliente se siente constreñido, con poca voluntad y sin libertad de expresar lo más significativo que le pasa ${ }^{(26)}$. El abordaje comprensivo del cliente como ser humano que posee conocimiento propio, resultante de su experiencia de vida, permite que el profesional de salud aprenda con él, existiendo un crecimiento personal y profesional, dejando de lado la posición de poseedor de todo el conocimiento.

A pesar de las presiones, algunas mujeres de este estudio no se someten pasivamente a las indicaciones que les son hechas, reaccionando con excusas a la hora de ejercer actividades para las que no están preparadas emocionalmente o reaccionando con actitudes de "burla" contra la situación por la que pasó.

Se percibe la utilización de una forma de poder que se expresa en las relaciones cotidianas, siendo ejercido por "unos" sobre "otros", configurando una micropolítica. El poder no se posee, se ejerce. Él se estructura en una red donde se encuentran puntos de resistencia y sumisión. El poder es una acción sobre acción, en el sentido de manipular la acción del otro o su campo de opciones ${ }^{(26)}$.

En lo que se refiere a orientaciones dirigidas a las puérperas, se utilizan otras estrategias como el refuerzo de la identidad materna, resultando común el que las puérperas sean llamadas rutinariamente como "madre", "mamaíta" como si todo lo que ella fue y es se perdió al hacerse madre, o sea, todas sus identidades (mujer, esposa, estudiante, trabajadora) desaparecieran en función de la maternidad. En todos los procedimientos y diálogos con ella esto se repite a pesar de que esa mujer tenga su identificación a través de su nombre escrito encima de su cama:

Al terminar el baño dice: "pronto, la mamaíta lo hizo todo derechito, aprendió derechito" (N.O).

"y ay mamaíta, ¿estás preparada? vamos a comenzar. Usted ya vio como se enreda. Lava la cara sólo con agua y después lava la cabecita con agua y jabón, vamos yo te voy a dar nota, aqui es un hospital escuela y la mamaíta tiene que salir de aquí sabiendo todo derechito" (N.O).

En el transcurso de la historia, la identidad femenina viene siendo definida básicamente por la maternidad. La secuencia niña-mujer-boda-maternidad es una secuencia naturalizada de acontecimientos que sobrepasan la vida de las mujeres, independientemente de su concreción en lo real o permanencia al nivel del imaginario. La mujer está condicionada desde la infancia para su futuro papel social de madre y todo su desarrollo es norteado por ese condicionamiento, incluso aunque ella nunca llegue a ser madre. Ser madre es un proceso culturalmente constituido que traspone el acto biológico de gestar y parir un hijo/hija y refleja el espacio social designado a la mujer en la sociedad ${ }^{(27)}$. 
Es necesario que al dirigirnos a las puérperas nos refiramos a ellas llamándolas por sus propios nombres, son, ante todo, mujeres. Llamarlas "madre", o "mamita", refuerza su identidad materna y esa actitud puede hacerle calar en su ser mujer que en esos momentos puede estar más necesitado de atención. Además, entendemos que la enfermería precisa mostrar respeto al cliente y muchas veces el simple hecho de llamar a la mujer madre por su nombre demuestra que ella existe como persona, con su identidad distinta de las demás mujeres.

\section{CONSIDERACIONES FINALES}

Consideramos que la mujer en la fase de puerperio-maternidad tiene necesidad de sentirse apoyada en sus miedos e inseguridades, posee también limitaciones emocionales y físicas que le dificultan la realización de los cuidados a su hijo.

El equipo de enfermería utiliza medios como el refuerzo de identidad materna, llamando a las mujeres madres como "mamita" en vez de llamarlas por el nombre, para hacerles ver su "deber" social metido en la maternidad y estas se convierten en prisioneras de un papel social que se define a través de los tiempos a partir del momento en que la mujer tiene un hijo. El problema es el respeto, tanto a la estructura de la maternidad como a la persistencia de los mitos que la cercan. El equipo de enfermería debe tomar conciencia de la fragilidad emocional que la mujer puérpera experimenta al hacerse madre, entender y respetar su angustia, fragilidad, sus miedos e inseguridades. Así, es necesario que veamos a la mujer madre/puérpera, ante todo como mujer, con sus deseos y virtudes que preceden su función materna.

Vale también resaltar que el precario proceso de comunicación que prevalece en el ámbito hospitalario dificulta la interacción de la puérpera con el equipo de enfermería reflejado en el proceso de aprendizaje de los cuidados al recién nacido tan importante en este periodo. Se hace necesario reflexionar sobre el método utilizado en educación en salud en el sistema de alojamiento conjunto de la institución en cuestión, pues este viene asumiendo fórmulas predefinidas, impositivas y así están siendo cargados los potenciales creativos y los conocimientos traídos por la mujer-madre no deparando beneficios para esta, pues no le deja espacio para que ejerza su maternidad de manera que crea conveniente. De igual modo, los funcionarios de enfermería del local no tienen oportunidad de desarrollar su potencial creativo para desarrollar/enseñar la técnica del baño, lo que puede transformar esa actividad en algo repetitivo, desgastante y estresante tanto para ella como para la puérpera a la que asiste.

La educación para la salud es esencial para la socialización del cuidado humano y de la familia. No existen recetas, guías de enseñanza o manuales para enseñarla. El cuidado técnico puede ser enseñado, pero el cuidar en sentido amplio, como proceso interactivo, precisa estar con el otro, ser vivido.

Cabe al equipo de enfermería, y en especial a la enfermera, volver al cuidado más humanizado, menos rutinario, apoyando la competencia de la mujer madre, de su equipo, haciéndose compañeros en la prestación de cuidados al recién nacido, resultando en la integración y aumento del vínculo afectivo madre-hijo, principales objetivos del sistema alojamiento conjunto. 


\section{REFERÊNCIAS}

1. Brasil. Portaria ํo. 1016, de 26 de agosto de 1993. Dispõe sobre as normas básicas de alojamento conjunto. Diário Oficial da União, Brasília, 01 set. 1993.

2. Soares, A. V. N.; Silva, I. A. Representações de puérperas sobre o sistema alojamento conjunto: do abandono ao acolhimento. Rev. Esc Enferm USP 2003; 37(2):72-80.

3. D'Avila, CGC; Gonçalves, R. O conhecimento de puérperas quanto ao cuidado com o coto umbilical: Considerações sobre a assistência de enfermagem. Rev Paul Enf 2003; 22 (1): 22-30 (Jan- Abr 2003).

4. Fonseca, LMM; Scochi, CGS; Mello, DF. Educação em saúde de puérperas em alojamento conjunto neonatal: aquisição de conhecimento mediado pelo uso de um jogo educativo. Rev Latino-Am Enf, mar/abr 2002, Vol 10, № 2, p.166-71. ISSN 0104-1169.

5. Minayo, MCS. O desafio do conhecimento: pesquisa qualitativa em saúde. Rio de Janeiro (RJ): ABRASCO; 1992.

6. Brasil. Ministério da Saúde. Diretrizes e normas regulamentadoras de pesquisas envolvendo seres humanos. Resolução 196/96 do Conselho nacional de saúde, de 10 de outubro de 1996.

7.Ferreira, ABH. Médio Dicionário Aurélio. Rio de Janeiro: Nova Fronteira (1980).

8. Maldonado, MT; Dickstein, J. Nós estamos grávidos. 8 ed. São Paulo: Saraiva, 1996.

9. Brazeton, TB. A dinâmica do bebê, Rio Grande do Sul: Ed. Artes Médicas, 1987. 10. Montagu, A. Tocar: O significado humano da pele. Trad. De Maria Silvia Mourão Netto. 4 ed.. São Paulo: Summus, 1988.

11. Gaíva, MAM; Gomes,MMF. Cuidando do Neonato: Uma abordagem de enfermagem. Goiânia: AB, 2003.

12. Kenner, C. Enfermagem Neonatal. 2 ed. Trad. De Neonatal Care. Rio de Janeiro: Reichmann \& Affonso, 2001.

13. São Paulo, Secretária do Estado da Saúde. Centro de Vigilância Epidemiológica das Infecções Hospitalares. Cuidados com o banho, coto umbilical e cateterização de vasos umbilicais. São Paulo (SP): Secretária de Estado da Saúde, 1993.

14. Soares, AVN; Silva, IA. Representações de puérperas sobre o sistema alojamento conjunto: do abandono ao acolhimento. Rev Esc Enf USP 2003; 37 (2): 72-80.

15. Foucalt, M. Vigiar e punir: nascimento da prisão. Trad Raquel Ramalhete. 13 ed. Petrópolis: Vozes, 1996.

16. Gasparetto, S; Bussab, SR. A necessidade de um trabalho preventivo em maternidade: instruções sobre o comportamento do recém-nascido. Faculdade de Saúde Pública - http://www.fsp.usp.br/BUSSAB.HTM - Acesso em 07/08/2000.

17. Maldonado, MT. As sementes do amor: Educar crianças de 0 a 3 anos para a paz. São Paulo: Editora Planeta do Brasil, 2003.

18. Santos, VCS; Prado, ML; Boehs, AE. Atuação da enfermeira junto ao casal/RN, no processo de parir, embasada na teoria de Madeleine Leininger. Texto \& Contexto Enf. 2002; 9 (2): 375-87 Florianópolis, 2000.

19. Zampieri, MFM. O processo educativo: interpretando o som da humanização. In: Oliveira, ME de; Zampieri, MFM; Brüggemann, OM. A Melodia da Humanização: Reflexões sobre o cuidado no processo do nascimento. Florianópolis: Cidades Futura, 2001. (142 Páginas)

20. Waldow, VR. Cuidado humano: resgate necessário. 2 ed. Porto Alegre: Sagra Luzzato, 1999. 
21. Waldow, VR. Cuidar/Cuidado: o domínio unificador da enfermagem. In: Waldow, VR; Lopes, MJM; Meyer, DE. Maneiras de cuidar, maneiras de ensinar, 1998. p.18. Porto Alegre: Artes Medicas, 1995.

22. Silva, MAR. Mulheres Em Conflito Com A Função Materna Na Internação Conjunta De Uma Unidade Pediátrica: Vivenciando o altruísmo. [dissertação] Cuiabá (MT): Programa Interinstitucional UFSC/UFMT; 1999.

23. Serrurier, C. Elogio às mães más. Trad. De Sônia Goldfeder. São Paulo: Summus, 1993.

24. Kitzinger, S. Mães: um estudo antropológico da maternidade. Lisboa: Presença, 1978.

25. Maldonado, MT; Canella, P. Recursos de relacionamento para profissionais de saúde: a boa comunicação com clientes e seus familiares em consultórios, ambulatórios e hospitais. Rio de Janeiro: Reichmann \& Affonso Editores, 2003.

26. D'Oliveira, AFPL. Gênero e violência nas práticas de saúde: Contribuição ao estudo da atenção integral à mulher. São Paulo, 1996. 193p. Dissertação (Mestrado) - Faculdade de Medicina, Universidade de São Paulo.

27. Grisci, CLI. Ser mãe: produção dele, reprodução dela. In: Cardoso, RS. (org.). É uma mulher... Petrópolis: Vozes, 1994. p.29-51. 